Rev. Adm. Saúde - Vol. 18, № 70, jan. - mar. 2018

http://dx.doi.org/10.23973/ras.70.85

ARTIGO DE REVISÃO

\title{
Cuidados paliativos em atenção domiciliar: uma revisão bibliográfica
}

Palliative care in home care: a bibliographic review

\author{
Gabriella Belém Vasconcelos ${ }^{1}$, Patrícia Mora Pereira ${ }^{2}$
}

1. Gerontóloga e especialista em administração hospitalar e sistemas de saúde. Aprimoranda do Programa de Estudos Avançados em Administração Hospitalar e Sistemas de Saúde do Hospital das Clínicas da Faculdade de Medicina da Universidade de São Paulo, (PROAHSA HCFMUSP), São Paulo, SP

2. Enfermeira e mestre em saúde pública. Aprimoranda do Programa de Estudos Avançados em Administração Hospitalar e Sistemas de Saúde do Hospital das Clínicas da Faculdade de Medicina da Universidade de São Paulo, (PROAHSA HCFMUSP), São Paulo, SP

\section{RESUMO}

Introdução: Os cuidados paliativos são definidos pela OMS como uma abordagem terapêutica que promove qualidade de vida e alívio do sofrimento aos pacientes e seus familiares que enfrentam problemas associados às doenças que ameaçam a continuidade da vida. A realização de cuidados paliativos em ambiente domiciliar favorece a aplicação dos princípios de cuidados paliativos, contribui para a redução da ansiedade do paciente e de seus familiares, possibilita o empoderamento da família no cuidado, além de colaborar para a redução da demanda por atendimentos hospitalares e dos custos em saúde em, aproximadamente, $50 \%$. Objetivos: Elaborar uma proposta de serviço de cuidados paliativos em atenção domiciliar. Método: Revisão bibliográfica e reuniões com especialistas em cuidados paliativos e operadoras de saúde. Resultados: Os resultados alcançados possibilitaram compreender o conceito da abordagem de cuidados paliativos e suas peculiaridades, além de identificar os recursos físicos e humanos necessários para o estabelecimento de um serviço de cuidados paliativos em atenção domiciliar. Conclusão: Uma equipe especializada e bem treinada pode ser considerada como a essência de um serviço de cuidados paliativos, assim a maior parte dos recursos financeiros devem ser aportadas inicialmente neste 
sentido. Além disso, apesar da existência de guias e manuais que auxiliam compreensão e estruturação de serviços desse tipo, ainda são necessárias adaptações nas ferramentas utilizadas para avaliação da performance paliativa, considerando o perfil de pacientes em internação domiciliar.

Palavras-chave: cuidados paliativos; assistência domiciliar; administração em saúde.

\section{ABSTRACT}

Introduction: Palliative care is defined by WHO as a therapeutic approach that promotes quality of life and relief of suffering for patients and their families facing problems associated with life-threatening diseases. Palliative care in the home environment favors the application of the principles of palliative care, contributes to reduce the anxiety of the patient and his / her family, enables the family to be empowered in care, besides contributes to reduce the demand for hospital and can decrease healthcare costs by approximately 50\%. Objective: To elaborate a proposal for a palliative care service in home care. Methods: The methods of bibliographic review and meetings with specialists in palliative care and health care providers was used. Results: The results obtained made it possible to understand the concept of the palliative care approach and its peculiarities, as well as to identify the physical and human resources needed to establish a palliative care service in homecare. Conclusion: $A$ well-trained and specialized team can be considered as the essence of a palliative care service, so most of the financial resources must be provided initially in this sense. In addition, although there are existing manuals and guides that help in understanding and structuring services of this type, it is still necessary to adapt the tools used to evaluate the palliative performance, considering the profile of patients in homecare programs.

Keywords: palliative care; home nursing; health administration.

\section{INTRODUÇÃO}

A Organização Mundial da Saúde ${ }^{1}$ (OMS) define cuidados paliativos como uma abordagem terapêutica que promove qualidade de vida e alívio do sofrimento aos pacientes e seus familiares que enfrentam problemas associados às doenças que ameaçam a continuidade da vida. Alguns princípios norteadores da prática em cuidados paliativos são destacados por Matsumoto ${ }^{2}$, sendo que estes foram revistos pela OMS em 2002. Assim, os princípios norteadores dessa abordagem são: 
- Promover o alívio da dor e de outros sintomas;

- Considerar a morte como um processo natural e afirmar a vida;

- Não acelerar nem adiar a morte dos pacientes;

- Integrar os aspectos psicológicos, emocionais e espirituais no cuidado ao paciente;

- Oferecer um sistema de suporte que possibilite ao paciente viver tão ativamente quanto possível até o momento da sua morte;

- Realizar o acolhimento aos familiares com o intuito de auxiliá-los durante o progresso da doença do paciente e o processo de luto;

- Oferecer abordagem multiprofissional com foco nas necessidades dos pacientes e seus familiares;

- Melhorar a qualidade de vida do paciente e influenciar positivamente o progresso da doença;

- Iniciar os cuidados paliativos o mais precocemente possível, juntamente com outras medidas de prolongamento da vida, ou seja, tratamentos curativos como quimioterapia e radioterapia, e incluir todas as investigações necessárias para melhor compreender e controlar situações clínicas estressantes.

Os cuidados paliativos ainda se encontram em processo de construção, motivo pelo qual a maior parte das estratégias de ação ainda são desafiadoras e requerem a atenção de uma equipe interdisciplinar. Por isso, essa abordagem não se restringe à mera execução de procedimentos em pacientes, mas à propagação da preocupação, interesse, interação e compromisso pelo cuidado ${ }^{3}$.

Os cuidados paliativos são realizados em cenários diversos, como em enfermarias hospitalares, hospices, instituições de longa permanência, ambulatórios especializados e em domicílio ${ }^{1,3,4}$. Entre esses, a modalidade focada neste artigo é a atenção domiciliar, ainda pouco praticada no Brasil, sobretudo no sistema de saúde suplementar.

A promoção de cuidados paliativos em ambiente domiciliar permite a pacientes idosos a possibilidade de continuar em seu contexto familiar e social, com atenção multiprofissional, especializada e disposta a oferecer suporte e orientação aos familiares e/ou cuidadores, evitando internações recorrentes e muitas vezes desnecessárias. Assim, o idoso permanece em um ambiente familiar, com riscos diminuídos e assistência integral, o que contribui para a melhorar e manutenção de sua qualidade de vida ${ }^{5}$. 
Em relação aos pacientes pediátricos, estes percebem o hospital como uma experiência misteriosa e aterrorizante, associando-o com um local estranho, de punição, tristeza e solidão, onde é proibido brincar. Para os pais, ter seu filho hospitalizado é percebido como falha em relação a maternagem, gerando sintomas de ansiedade e culpa. Assim, cuidar de pacientes pediátricos em ambiente domiciliar pode diminuir a ansiedade do paciente e seus familiares, possibilitar o empoderamento dos familiares no cuidado, facilitar o uso de ludoterapia e a utilização de brinquedos terapêuticos e promover e/ou reestabelecer o vínculo afetivo emocional entre pais e filhos ${ }^{6,7}$.

Uma estimativa da OMS (apud Bomfim) 8 mostra que, no Brasil, entre 521 mil e 536 mil pessoas necessitam de cuidado paliativo, no entanto, esses tendem a ser indicados somente na fase de final de vida, restringindo a atuação das equipes especializadas nesta área ${ }^{1}$. Neste cenário, uma publicação feita em 2015 pelo jornal The Economist ${ }^{9}$ mostra o Brasil em 42이 lugar em um ranking que mostra a qualidade de morte entre os países ao redor do mundo, cujo primeiro lugar é ocupado pelo Reino Unido, com Austrália em segundo e Nova Zelândia em terceiro. O Brasil ocupa o fim da lista, próximo a países como Hungria, México, Tailândia e Venezuela.

Do mesmo modo, o Atlas Global de Cuidados Paliativos no Fim da Vida (Global Atlas of Palliative Care at the End of Life), publicado em 2014 pela OMS em conjunto com a Worldwide Palliative Care Alliance (WPCA), mostra o Brasil entre um grupo de países que apresentam o desenvolvimento de iniciativas em cuidados paliativos ainda muito isoladas e focadas em áreas que oferecem maior apoio localmente, como em grandes centros urbanos com recursos de profissionais de saúde, materiais e medicamentos mais abundantes e de acesso mais facilitado ${ }^{10}$.

Uma pesquisa realizada por Othero (apud Gomes e Othero) ${ }^{11}$ identificou que quase metade dos serviços de Cuidados Paliativos do Brasil atuam no estado de São Paulo, com predominância de atendimento tipo ambulatorial, atendendo pacientes oncológicos e não oncológicos, adultos e idosos, em sua maioria com financiamento público. Isso demonstra a escassez de serviços específicos para a modalidade de atenção domiciliar, sobretudo particulares, mesmo a morte em casa sendo apontada como preferencial para pacientes e familiares em pesquisa realizada em 2017 pela Kaiser Family Foundation em parceria com o jornal The Economist.

De acordo com Fripp ${ }^{12}$, a assistência em ambiente domiciliar com o apoio da família favorece a aplicação dos princípios de cuidados paliativos, integram aspectos psicossociais e espirituais, e dão suporte para que a família possa auxiliar nos cuidados à doença e trabalhar o luto e a perda.

Além disso, o cuidado em ambiente domiciliar permite o aumento da qualidade do cuidado, bem como, contribui para reduzir a demanda por atendimentos hospitalares, o tempo médio de permanência na internação, os riscos de infecção hospitalar, principalmente, as infecções cruzadas e os custos associados a estas situações. 
Assim, o objetivo deste artigo foi elaborar uma proposta de serviço de cuidados paliativos em atenção domiciliar. Como objetivos específicos foram definidos:

- Identificar os recursos físicos necessários para a abordagem terapêutica em cuidados paliativos;

- Identificar os recursos humanos necessários para uma atenção integral ao paciente em cuidados paliativos;

- Identificar os momentos de atuação, bem como, os principais instrumentos utilizados na abordagem em cuidados paliativos.

\section{MÉTODOS}

Para levantamento de informações sobre cuidados paliativos em atenção domiciliar, foi utilizado o método de revisão bibliográfica. Os documentos principais sob os quais esta pesquisa de baseou foram o guia "Planning and implementing palliative care services: a guide for programme managers", publicado em 2016 pela Organização Mundial da Saúde; o guia "Vamos falar de cuidados paliativos", publicado em 2015 pela Sociedade Brasileira de Geriatria e Gerontologia (SBGG); o "Manual de cuidados paliativos", publicado pela Academia Nacional de Cuidados Paliativos (ANCP) em 2009; e o livro "Cuidado paliativo", publicado em 2008 pelo Conselho Regional de Medicina do Estado de São Paulo (CREMESP).

Além dos materiais citados, foram realizadas buscas nas bases de dados Scientific Eletronic Library Online (SciELO), PubMed, LILACS e Google Acadêmico, utilizando os descritores "cuidados paliativos", "atenção domiciliar" e "cuidados paliativos" e "atenção domiciliar", com a intenção de encontrar artigos científicos que relacionassem ambos os temas.

Ademais, para validar as informações alcançadas por meio da revisão bibliográfica, realizou-se uma reunião com uma enfermeira especialista em cuidados paliativos, bem como reuniões com quatro operadoras de saúde de grande atuação na cidade de São Paulo, SP.

\section{RESULTADOS}

Os resultados encontrados são apresentados nos próximos tópicos de modo a elucidar os principais conceitos encontrados na literatura estudada, e validadas nas reuniões realizadas.

O primeiro tópico consta sobre a abordagem de cuidados paliativos, seus principais momentos e ferramentas indicadas para acompanhamento dos sintomas e evolução da doença. O tópico seguinte versa sobre os cuidados paliativos realizados em ambiente domiciliar, os recursos físicos e humanos necessários para implantação de um serviço de paliativos nesse contexto. 


\section{Cuidados paliativos}

De acordo com Menezes ${ }^{13}$, o modelo de morte construído pela sociedade ocidental contemporânea se construiu ao longo do século XX, com todas as transformações tecnológicas ocorridas no campo da medicina, devido principalmente às duas grandes guerras mundiais ocorridas neste período. Esse avanço tecnológico viabilizou o prolongamento da vida de diferentes maneiras e levou a morte para dentro do hospital, monitorada e controlada por médicos e outros profissionais da saúde, silenciosa, escondida, inconsciente e, por vezes, solitária.

Nesse cenário, a OMS adota, em 1990, "a filosofia dos cuidados paliativos como uma terapêutica humanizada ao cuidado de pacientes cuja doença não responde ao tratamento curativo, sobretudo, quando [...] encontra-se em fase avançada e progressiva" ${ }^{3}$. Esse conceito foi revisado novamente pela OMS em 2002 e, atualmente, os cuidados paliativos são considerados como uma abordagem terapêutica que visa promover qualidade de vida e minimizar o sofrimento de pacientes e seus familiares e/ou cuidadores que enfrentam desafios relacionados enfermidades potencialmente ameaçadoras da continuidade da vida ${ }^{1}$.

O modelo estigmatizado de cuidados paliativos como sinônimo de terminalidade se deve ao fato de que este, inicialmente, era centrado na assistência a pacientes em sua fase final de vida. No entanto, este conceito foi expandido e, atualmente, considera-se que os cuidados paliativos devem ser oferecidos a pacientes e seus familiares o mais precocemente possível, possibilitando seu acompanhamento durante todo o processo da doença potencialmente ameaçadora de vida, desde seu diagnóstico até o processo de luto ${ }^{2,14}$, considerando sempre o foco da atenção não na doença a ser curada, mas no paciente em seus aspectos biopsicossociais, compreendido como "um ser biográfico, ativo, com direito a informação e autonomia plena para as decisões a respeito de seu tratamento"15.

A aplicação da abordagem de cuidados paliativos desde o momento do diagnóstico implica uma variação na necessidade de cuidados no decorrer da doença e, posteriormente, no processo de luto. Ao longo desse percurso, a premência de cuidados se mostra maior logo no início, onde é preciso acolher e orientar pacientes, familiares e/ou cuidadores quanto à doença, diminuindo $\mathrm{a}$ ansiedade; seguida por períodos de exacerbação, normalmente em momentos nos quais o paciente apresenta sintomas agudos; alcança estabilidade conforme a doença passa a se comportar de forma crônica e cresce gradualmente, de acordo com o aumento da debilidade do paciente. A necessidade de cuidados paliativos alcança seu ápice nos últimos dias/horas de vida e no momento da morte, sendo diminuída após esse evento, conforme a construção do processo de luto ${ }^{14}$.

\section{Momentos em cuidados paliativos e os principais instrumentos utilizados}


De acordo com a Sociedade Brasileira de Geriatria e Gerontologia ${ }^{14}$, a abordagem paliativa apresenta benefícios potenciais desde a fase prédiagnóstica, embora os cuidados paliativos em si sejam iniciados apenas a partir do diagnóstico da doença. Nesses dois momentos, o paciente e seus familiares e/ou cuidadores recebem informações acerca da doença e sobre o que esperar de sua evolução, dos sistemas de saúde e da equipe assistencial, iniciando um vínculo que será muito importante nas fases posteriores.

É importante ressaltar que, neste momento, a abordagem de cuidados paliativos segue em conjunto com as terapias redutoras de risco e com o tratamento modificador da doença. A necessidade de cuidados paliativos aumenta de acordo com a diminuição das alternativas terapêuticas de caráter curativo disponíveis e a aproximação da terminalidade, assim, "o foco e os objetivos do cuidado vão progressivamente se deslocando desde uma ênfase em tratamentos modificadores da doença até tratamentos com intenção exclusivamente paliativa"14.

Dessa forma, os cuidados paliativos podem ser divididos em cinco momentos: pré-diagnóstico, diagnóstico, cuidados ao fim da vida / terminalidade, últimas horas de vida e cuidados no processo de luto.

Por meio da aplicação da Escala de Performance Paliativa (Palliative Performance Scale - PPS), é possível obter indicações de sobrevida do paciente e, assim, acompanhar a evolução da doença e a necessidade de iniciar os cuidados ao fim de vida (Tabela 1). O PPS é uma adaptação da escala de performance de status de Karnofsky, amplamente utilizada para avaliação de pacientes oncológicos com o objetivo de classificar sua capacidade em realizar atividades básicas de vida diária e documentar o seu declínio ${ }^{12,16}$.

A escala PPS indica níveis que variam de 0 a 100 em porcentagem e, de acordo com um estudo realizado por Harlos (apud Maciel) ${ }^{15}, 90 \%$ dos pacientes classificados com PPS igual a 50\% apresentam sobrevida de apenas seis meses e pacientes em final de final apresentam $20 \%$ de performance paliativa, ou seja, indicam sinais de terminalidade. Na maior parte desses casos, o tratamento curativo já não apresenta grandes benefícios e, por efeito da doença, o sofrimento se manifesta de moderado a intenso, sendo os cuidados paliativos indicados com o propósito de atuar na manutenção de conforto, alívio do sofrimento e dignidade da vida ${ }^{15,16}$.

Tabela 1. Escala de Performance Paliativa (PPS).

\begin{tabular}{llllll}
\hline$\%$ & Deambulação & $\begin{array}{l}\text { Atividade e } \\
\text { evidência de } \\
\text { doença }\end{array}$ & Autocuidado & Ingestão & $\begin{array}{l}\text { Nível de } \\
\text { consciência }\end{array}$ \\
\hline 10 & Completa & $\begin{array}{l}\text { Normal, sem } \\
\text { evidência de } \\
\text { doença }\end{array}$ & Completo & Normal & Completo \\
0 & & & &
\end{tabular}




\begin{tabular}{|c|c|c|c|c|c|}
\hline 90 & Completa & $\begin{array}{l}\text { Normal, alguma } \\
\text { evidência de } \\
\text { doença }\end{array}$ & Completo & Normal & Completo \\
\hline 80 & Completa & $\begin{array}{l}\text { Com esforço, } \\
\text { alguma } \\
\text { evidência de } \\
\text { doença }\end{array}$ & Completo & Normal & Completo \\
\hline 70 & Reduzida & $\begin{array}{l}\text { Incapaz para o } \\
\text { trabalho, alguma } \\
\text { evidência de } \\
\text { doença }\end{array}$ & Completo & $\begin{array}{l}\text { Normal ou } \\
\text { reduzida }\end{array}$ & Completo \\
\hline 60 & Reduzia & $\begin{array}{l}\text { Incapaz de } \\
\text { realizar hobbies, } \\
\text { doença } \\
\text { significativa }\end{array}$ & $\begin{array}{l}\text { Assistência } \\
\text { ocasional }\end{array}$ & $\begin{array}{l}\text { Normal ou } \\
\text { reduzida }\end{array}$ & $\begin{array}{l}\text { Completo ou } \\
\text { com períodos } \\
\text { de confusão }\end{array}$ \\
\hline 50 & $\begin{array}{l}\text { Sentado ou } \\
\text { deitado }\end{array}$ & $\begin{array}{l}\text { Incapacitado } \\
\text { para qualquer } \\
\text { trabalho, doença } \\
\text { extensa }\end{array}$ & $\begin{array}{l}\text { Assistência } \\
\text { considerável }\end{array}$ & $\begin{array}{l}\text { Normal ou } \\
\text { reduzida }\end{array}$ & $\begin{array}{l}\text { Completo ou } \\
\text { com períodos } \\
\text { de confusão }\end{array}$ \\
\hline 40 & Acamado & Idem & $\begin{array}{l}\text { Assistência } \\
\text { quase } \\
\text { completa }\end{array}$ & $\begin{array}{l}\text { Normal ou } \\
\text { reduzida }\end{array}$ & $\begin{array}{l}\text { Completo ou } \\
\text { com períodos } \\
\text { de confusão }\end{array}$ \\
\hline 30 & Acamado & Idem & $\begin{array}{l}\text { Dependência } \\
\text { completa }\end{array}$ & Reduzida & $\begin{array}{l}\text { Completo ou } \\
\text { com períodos } \\
\text { de confusão }\end{array}$ \\
\hline 20 & Acamado & Idem & Idem & $\begin{array}{l}\text { Ingestão } \\
\text { limitada a } \\
\text { colheradas }\end{array}$ & $\begin{array}{l}\text { Completo ou } \\
\text { com períodos } \\
\text { de confusão }\end{array}$ \\
\hline 10 & Acamado & Idem & Idem & $\begin{array}{l}\text { Cuidados } \\
\text { com a boca }\end{array}$ & $\begin{array}{l}\text { Confuso ou em } \\
\text { coma }\end{array}$ \\
\hline 0 & Morte & - & - & - & - \\
\hline
\end{tabular}

Fonte: Arantes, 2009, p. 25, adaptado pela autora.

De acordo com Maciel ${ }^{15}$, a iniciação precoce da abordagem paliativa possibilita antever e prevenir sintomas. O medo de manifestar sintomas como dor e dispneia e não alcançar o conforto necessário é associado à não aceitação por parte do paciente e da família pela assistência em ambiente domiciliar. Assim, além do acompanhamento da performance paliativa, a percepção de sinais e o manejo correto dos sintomas é imprescindível.

Dessa forma, o controle dos sintomas em cuidados paliativos, se baseia nos princípios descritos a seguir:

- Avaliar antes de tratar; 
- Explicar as causas dos sintomas;

- Não esperar que um doente se queixe;

- Adotar uma estratégia terapêutica mista;

- Monitorizar os sintomas;

- Reavaliar regularmente as medidas terapêuticas;

- Cuidar dos detalhes;

- Estar disponível ${ }^{15}$.

Para auxiliar nesse processo, a Escala de Avaliação de Sintomas de Edmonton (Edmonton Symptom Assessment Scale - ESAS) foi elaborada no Canadá pelo serviço de Cuidados Paliativos de Edmonton e adaptada por Neto (apud Maciel) ${ }^{15}$. Trata-se de um questionário auto referido e simples que visa indicar sintomas objetivos e subjetivos. Nessa escala, o paciente ou seu cuidador/familiar atribui uma nota de 0 a 10 para cada sintoma, sendo 0 a ausência e 10 a maior intensidade do sintoma (Quadro 1). A ESAS pode ser aplicada por profissionais médicos e/ou enfermeiros, contribuindo para a tomada de decisão e oferecendo subsídios para definição de ações e condutas de alívio aos sintomas evidenciados ${ }^{12,15,17}$.

Quadro 1. Escala de Avaliação de Sintomas de Edmonton (ESAS). 


\begin{tabular}{|c|c|c|}
\hline \multicolumn{3}{|c|}{ AVALIAÇĀO DE SINTOMAS } \\
\hline \multicolumn{3}{|c|}{$\begin{array}{l}\text { Data: } \ldots \text { Preenchido por: } \\
\text { Por favor circule o } n^{\circ} \text {. que melhor descreve a intensidade dos seguintes sintomas neste } \\
\text { momento. (Também se pode perguntar a média durante as últimas } 24 \text { horas). }\end{array}$} \\
\hline Sem Dor & $=0-1-2-3-4-5-6-7-8-9-10=$ & $=$ Pior dor possivel \\
\hline Sem Cansaço & $=0-1-2-3-4-5-6-7-8-9-10=$ & $=$ Pior cansaço possivel \\
\hline Sem Náusea & $=0-1-2-3-4-5-6-7-8-9-10=$ & $=$ Pior náusea possivel \\
\hline Sem Depressão & $=0-1-2-3-4-5-6-7-8-9-10=$ & $=$ Pior depressäo possivel \\
\hline Sem Ansiedade & $=0-1-2-3-4-5-6-7-8-9-10=$ & $=$ Pior ansiedade possivel \\
\hline Sem Sonolência & $=0-1-2-3-4-5-6-7-8-9-10=$ & $=$ Pior sonolência possivel \\
\hline Muito Bom Apetite & $=0-1-2-3-4-5-6-7-8-9-10=$ & $=$ Pior apetite possivel \\
\hline Sem Falta de Ar & $=0-1-2-3-4-5-6-7-8-9-10=$ & $=$ Pior falta de ar possivel \\
\hline $\begin{array}{l}\text { Melhor sensação } \\
\text { de bem estar }\end{array}$ & $=0-1-2-3-4-5-6-7-8-9-10=$ & $=\begin{array}{l}\text { Pior sensação de } \\
\text { bem estar possivivel }\end{array}$ \\
\hline
\end{tabular}

Fonte: MACIEL, 2008, p. 27.

\section{Últimas 48 horas}

Segundo Américo ${ }^{18}$ "as últimas horas do paciente em fase final de vida são a continuidade da evolução progressiva de sinais e sintomas". A atenção nessa fase deve ser contínua e toda equipe precisa estar apta a observar e alertar sobre mudanças no quadro clínico do paciente, que se apresenta em declínio funcional avançado e irreversível.

Nesse momento, a atenção da equipe de cuidados paliativos deve se voltar para o controle dos novos sintomas, que devem ser tratados e antevistos, com vistas a identificar a necessidade ou não de sedação paliativa, bem como para o preparo do paciente e de seus familiares e/ou cuidadores para os eventos futuros ${ }^{18,19}$.

A equipe deve permitir que o paciente se sinta devidamente acolhido em seu ambiente, providenciado a presença contínua de um familiar ou cuidador ao seu lado. A individualidade deve ser respeitada, assim como a angústia, os medos e a tristeza dos familiares e/ou cuidadores não podem ser 
desconsiderados ou medicados. Caso solicitado, a equipe pode auxiliar no contato com um capelão ou assistente espiritual de confiança para conforto da família, sempre considerando sua espiritualidade e suas crenças religiosas ${ }^{18}$.

\section{Luto}

Mesmo após a morte do paciente, os cuidados paliativos devem prosseguir com o acolhimento e assistência ao processo de luto de familiares e/ou cuidadores pela equipe multiprofissional ${ }^{20}$. De acordo com a OMS ${ }^{1}$, o suporte e o cuidado no luto também são componentes cruciais dos cuidados paliativos, pois pacientes e seus familiares frequentemente apresentam necessidade de apoio emocional para enfrentar as perdas associadas à morte e ao luto, $\mathrm{e}$ algumas famílias enlutadas desenvolvem dores complexas que demandam tratamento e um acompanhamento psicológico mais específico.

Além do suporte psicológico e acompanhamento terapêutico, a assistência social possui um papel importante no período de luto, sendo responsável por fornecer orientações quanto aos trâmites burocráticos relacionados à morte recente do paciente, como questões ligadas a seguro de vida, pensões, heranças e outros, auxiliando na reorganização familiar após o óbito.

\section{Cuidado paliativo em ambiente domiciliar}

Para a implantação de um serviço de cuidados paliativos em atenção domiciliar, a OMS ${ }^{1}$ indica 8 passos:

1. Avaliar necessidades dos pacientes e recursos disponíveis;

2. Estabelecer formalização da organização por meio de termos de referência e registro com autoridades;

3. Criar um plano de ação (quais recursos serão necessários, como podese obtê-los, público alvo e serviços que serão cobertos);

4. Recrutar e desenvolver um programa de treinamento contínuo;

5. Mobilizar recursos;

6. Integrar dentro do sistema de saúde, associando com a atenção primária e a terciária de referência da operadora;

7. Divulgar o serviço;

8. Encorajar a participação de associações, grupos e estudantes. 
Em cuidados pediátricos é necessário, além destes passos, adequar para o cuidado paliativo em pediatria e revisar a legislação, incluindo a proteção e os direitos da criança ${ }^{1}$. O mesmo é indicado em casos de cuidado a idosos, mantendo a equipe atualizada quanto às principais legislações no que tange à essa faixa etária, sendo elas a Política Nacional do Idoso e o Estatuto do Idoso.

Ainda, a OMS ${ }^{1}$ recomenda que se inicie o serviço focado em pacientes com câncer terminal, pois mais de $80 \%$ desses pacientes sofrem com dores severas e outros sintomas que necessitam de alívio e conforto imediato e depois, gradualmente, expanda o serviço para doenças crônicas e outras doenças com tempo de vida limitado.

Pacientes que necessitam de cuidados paliativos podem necessitar de cuidados de complexidades distintas, o que demanda integração entre os níveis de atenção. Por isso, é preciso articular os diferentes componentes do sistema de saúde, criando um mecanismo de referência e contra referência para casos de intercorrência clínica ${ }^{1}$.

\section{Recursos físicos}

É necessário que o ambiente seja seguro e acessível para que a equipe multiprofissional de cuidados paliativos possa planejar e prestar a sua assistência. Nos casos de equipamentos de suporte à vida que funcionam à base de energia elétrica, é importante disponibilizar geradores e/ou entrar em contato com a companhia responsável pela distribuição de energia elétrica na região, explicando o caso para prevenir as chances de cortes no fornecimento.

O local também precisa ser limpo, arejado e com entrada de luz natural, de forma e evitar possíveis infecções. É preciso ter saneamento básico e coleta de lixo, sendo o material perfurocortante recolhido pelo serviço de atenção domiciliar para o descarte correto.

Além das questões de estrutura física, também é necessário analisar a estrutura domiciliar no momento de avaliação do paciente, facilitando a classificação do ambiente de cuidado. Tais observações podem ser feitas pelo assistente social da equipe multidisciplinar. É necessário também que o local respeite as normas propostas pela ANVISA na RDC nำ50/2002.

A partir da leitura dos materiais levantados e das informações obtidas por meio das reuniões realizadas com especialistas em cuidados paliativos e operadoras de saúde foi possível identificar que, do ponto de vista de uma empresa que deseja implantar um serviço de cuidados paliativos domiciliar, é importante considerar os pontos seguintes como parte da infraestrutura mínima necessária para início das atividades:

- Central de atendimento e operações 24 horas: disponível para assistir a família e o paciente no caso de necessidades, bem como, a equipe multiprofissional atuante na casa e as operadoras de saúde. 
- Farmácia 24 horas: dispensação de medicamentos em casos de urgência e emergência e quando não for possível controlar os sintomas com os medicamentos já disponibilizados em domicílio.

- Transporte: deslocar os profissionais de saúde e os materiais e medicamentos até os domicílios dos pacientes.

- Meios de comunicação: meio de comunicação entre os profissionais de saúde, pacientes e a central de atendimento e operações como, por exemplo, o uso de celulares ou tablets com conexão de videoconferência.

- Equipe multiprofissional: médicos, enfermeiros, auxiliar de enfermagem, psicólogo, fisioterapeuta e assistente social compõem a equipe básica. Caso o serviço deseje e a família concorde, também pode ser aberta a possibilidade de visitas conjuntas com residentes médicos ou multiprofissionais na área de cuidados paliativos visando colaborar com o aprendizado dos futuros profissionais desta especialidade. Também é possível incluir na equipe a presença de profissionais que atuem em terapias complementares como acupunturistas, massagistas e musicoterapeutas e, quando os cuidados forem destinados à pediatria, a presença de um pedagogo.

- Kit de atenção domiciliar: medicações e materiais médico-hospitalares, com o registro diário da quantidade utilizada e restante; equipamentos e documentação. Esse último é composto por:

1. Contrato de prestação de serviços

2. Termo de aceite de cuidados paliativos

3. Termo de não ressuscitação cardiopulmonar

4. Prontuário clínico contendo histórico anterior à implantação do serviço, anamnese, diagnóstico médico, diagnóstico de enfermagem com a utilização da sistematização da assistência de enfermagem (SAE), prescrição medicamentosa, anotações de enfermagem, evolução médica e da equipe multiprofissional, PPS, ESAS, escalas para avaliação de sintomas específicos e gráfico de sinais vitais.

Os suprimentos que serão necessários dependem muito da patologia e das condições clínicas do paciente que será atendido, entretanto, podemos citar alguns equipamentos e suprimentos básicos elencados pela OMS ${ }^{1}$ :

- Equipamentos: estetoscópio (responsabilidade do profissional); esfigmomanômetro (responsabilidade do profissional); termômetro; abaixador de língua; pinça para a realização de curativo. 
- Equipamentos de suporte: colchão de ar ou água ou casca de ovo; aparelho de sucção; nebulizador; cilindro de oxigênio; oxímetro.

- Suprimentos: tesoura; algodão; gaze; bandagem e fita micropore; luvas; equipo de soro; cateter venoso central; seringas e agulhas; caixa coletora de perfurocortante; cateter de sucção; cateter urinário; bolsa coletora de urina; sonda nasogástrica e nasoenteral; álcool.

Como principais medicamentos sugeridos pela $\mathrm{OMS}^{1}$ para o controle de sintomas podemos citar:

- Controle da dor: paracetamol; diclofenaco; fosfato de codeína; tramadol; morfina e gabapentina.

- Controle de sintomas gastrointestinais: metoclopramida; domperidona; dexametasona; ranitidina; lorapamida; sais de hidratação oral e bisacodil.

- Tratamento de Ferida: peróxido de hidrogênio e pomada betadine.

- Tratamento de sintomas psicológicos: diazepam; haloperidol e amitriptilina.

- Antibióticos e Antifúngicos: ciprofloxaceno; metronidazol; amoxicilina e fluconazol;

- Suplementos: suplementos com alto ganho calórico e proteico; ferro; vitaminas e minerais.

Vale lembrar que, apesar de os equipamentos, materiais e medicamentos supracitados serem indicados pela OMS, outros itens podem ser adicionados ou retirados dessa lista. Esse ponto varia de acordo com a disponibilidade local, a política da empresa de atenção domiciliar e as necessidades do paciente.

Além disso, é importante ressaltar que, conforme dito anteriormente, uma das principais causas que levam ao paciente optar por não se tratar em casa é o medo de não ser possível controlar os sintomas, principalmente, a dor e a dispneia. Assim, a avaliação e o controle dos sintomas deve ser o ponto principal de atuação dos profissionais de cuidados paliativos em atenção domiciliar, de forma que o manejo dos sintomas seja adequado e evite a reinternação do paciente em hospitais de transição, hospices ou hospitais terciários.

\section{Recursos humanos}


A equipe de cuidados paliativos deve possuir habilidades de manejo de complicações, sejam estas oriundas da doença ou do tratamento; habilidades para manejo da dor e outros sintomas; abordagem centrada no paciente e em seus familiares, capacidade de prover acolhimento e humanização no ambiente de cuidado e propiciar cuidado biopsicossocial aos pacientes e seus familiares no momento da morte e do luto ${ }^{1,15}$.

Também é imprescindível possuir habilidades de comunicação, tomada de decisão de forma rápida e resolutiva e compreensão de outras necessidades como as espirituais. Compreender esses papéis e responsabilidade ajuda no funcionamento das equipes, a avaliar as necessidades de treinamento, bem como, permite que o cuidado ao paciente ocorra de forma integral e holística ${ }^{1}$.

Além disso, as decisões precisam ser tomadas em conjunto e é necessário apoio e respeito mútuo entre os profissionais. Reconhecer e verbalizar a importância das diferentes contribuições dos membros da equipe é essencial, bem como, reconhecer os limites de cada membro da equipe, de forma a evitar uma possível sobrecarga emocional, suportando uns aos outros em suas necessidades ${ }^{21}$.

Para iniciar seu funcionamento, um serviço de cuidados paliativos em atenção domiciliar deve contar com, no mínimo, dois enfermeiros que atuariam em escala de 12 horas de trabalho para 36 horas de descanso e, atuando em meio período, um médico, um psicólogo e um assistente social. Apesar disso, uma equipe ideal de cuidados paliativos deve contar com o suporte de toda uma equipe multidisciplinar, composta por técnicos/auxiliares de enfermagem, fisioterapeutas, fonoaudiólogos, nutricionistas, terapeutas ocupacionais, farmacêuticos, gerontólogos, nutrólogos e profissionais de práticas complementares, caso necessário ${ }^{1,3,15}$.

Além disso, é importante identificar familiares que possam auxiliar no cuidado diário. Esses devem ser devidamente treinados pela equipe responsável pelo paciente para identificar necessidades físicas, psicológicas e espirituais do paciente e contribuir para cuidados básicos, de forma a empoderar a família para o cuidado do seu familiar. Familiares que atuam ativamente no cuidado se sentem mais satisfeitos, porém estes também precisam de suporte e acolhimento pela equipe de cuidados paliativos ${ }^{1}$.

\section{CONSIDERAÇÕES FINAIS}

Apesar de ser ainda pouco difundida no Brasil, a abordagem de cuidados paliativos vem ganhando cada vez mais espaço no país, principalmente em grandes centros urbanos como a capital paulista.

As transições demográfica e epidemiológica vividas atualmente, juntamente com outros fatores como o avanço das tecnologias médicas e o modelo de pagamento por procedimento (fee for service) praticado atualmente na área da saúde contribuem, cada um à sua maneira, para o aumento dos custos dos sistemas de saúde público e privado. Nesse cenário, comumente são 
identificadas situações nas quais internações e procedimentos desnecessários são realizados na tentativa de prolongar a vida o máximo possível e afastar a morte às custas de prejuízos à qualidade de vida de pacientes com doenças potencialmente ameaçadoras da vida e incapacitantes.

Isso posto, os cuidados paliativos se apresentam como uma necessidade, contribuindo para a promoção de alívio e conforto a pacientes e seus familiares. Fazê-lo em domicílio traz benefícios quanto à segurança, comodidade, preservação da autonomia e empoderamento desses, mesmo em um momento tão delicado. Para que tudo isso ocorra, uma equipe especializada e bem treinada é imprescindível e é onde a maior parte dos recursos iniciais para a criação de um serviço de cuidados paliativos em atenção domiciliar deve estar. A equipe é responsável por acolher, orientar, assistir e amparar pacientes, familiares e cuidadores ao longo de todo o processo de paliativos, desde o diagnóstico até o processo de luto.

Ainda, é importante ressaltar a necessidade de se conhecer bem a empresa onde se deseja instituir o serviço, o perfil epidemiológico e socioeconômico de seus pacientes, bem como os principais parceiros que poderão contribuir para a criação do serviço. Por meio desse estudo, também foi possível observar que, apesar dos manuais e guias existentes que auxiliam na compreensão e estruturação de serviços desse tipo, ainda são necessárias adaptações nas ferramentas utilizadas para avaliação da performance paliativa, considerando o perfil de pacientes em internação domiciliar.

\section{REFERÊNCIAS}

1. World Health Organization (WHO). Planning and implementing palliative care services: a guide for programme managers. Suíça, 2016. Disponível em: < http://apps. who.int/iris/bitstream/10665/250584/1/9789241565417eng.pdf>. Acesso em: 19 abr. 2017.

2. Matsumoto DY. Cuidados paliativos: conceito, fundamentos e princípios. In: Manual de cuidados paliativos. Academia Nacional de Cuidados Paliativos ANCP. Rio de Janeiroi: Diagraphic, 2009.

3. Andrade CG, Costa SFG, Costa ICP.; et al. Cuidados paliativos e comunicação: estudo com profissionais de saúde do serviço de atenção domiciliar. Rev Fund Care Online. v.9, n.1, p.215-221, jan/mar 2017. DOI: http://dx.doi.org/10.9789/2175-5361.2017.v9i1.215-221.

4. Kaiser Family Foundation e The Economist. Four country survey on aging and end-of-life medical care. Abril, 2017. Disponível em: < http://www.kff.org/other/report/views-and-experiences-with-end-of-lifemedical-care-in-japan-italy-the-united-states-and-brazil-a-cross-countrysurvey/>. Acesso em: 06 jun. 2017. 
5. Faller JW, Brusnicki PH, Zilly A, Brofman, MCBFS, Cavalhieri L. Perfil de idosos acometidos por câncer em cuidados paliativos em domicílio. Rev. Kairós Gerontologia. v.19, n.22, p.22-43. São Paulo, 2016. Disponível em: <https://revistas.pucsp.br/index.php/kairos/article/view/31612/22012>. Acesso em: 05 jun. 2016.

6. Pettengill MAM, Ribeiro CA, Borba RIH. O cuidado centrado na criança e sua família: uma perspectiva para a atuação do enfermeiro pediatra. In: ALMEIDA F. A.; SABATÉS A. L. (Org). Enfermagem Pediátrica: a criança, o adolescente e a sua família no hospital. São Paulo: Manole, 2008.

7. Vasconcelos GB, Pereira PM. Serviço de cuidados paliativos em atenção domiciliar: manual de implantação. Trabalho de Estágio (Programa de Estudo Avançados em Administração Hospitalar e Sistemas de Saúde PROAHSA) - Programa de Aprimoramento Profissional do Hospital das Clínicas da Faculdade de Medicina da Universidade de São Paulo. Material não publicado. São Paulo, 2017.

8. Bomfim M. Cuidado paliativo na saúde avança no Brasil, mas ainda é isolado. Folha de São Paulo. Folha digital, postado às $12 \mathrm{~h}$ do dia $31 \mathrm{de}$ março de 2014. Disponível em:

<http://www1.folha.uol.com.br/seminariosfolha/2014/03/1432699-cuidadopaliativo-na-saude-avanca-no-brasil-mas-ainda-e-isolado.shtml>. Acesso em: 18 jun. 2017.

9. The Economist Intelligence Unit, commissioned by the Lien Foundation. The 2015 quality of death index ranking palliative care across the world: key findings infographic. Disponível em: <http://www.kff.org/other/report/viewsand-experiences-with-end-of-life-medical-care-in-japan-italy-the-unitedstates-and-brazil-a-cross-country-survey/>. Acesso em: 9 mai. 2017.

10.World Health Organization (WHO); WORLDWHIDE Palliative Care Alliance (WPCA). Global atlas of palliative care at the end of life. Disponível em: $<\mathrm{http}: / /$ www.who.int/nmh/Global_Atlas_of_Palliative_Care.pdf >. Acesso em: 9 mai. 2017.

11. Gomes ALZ, Othero MB. Cuidados paliativos. Estud. av., São Paulo, v. 30, n. 88, p. 155-166, Dec. 2016. Disponível em: <http://www.scielo.br/scielo.php?script=sci_arttext\&pid=S0103$40142016000300155 \&$ Ing=en\&nrm=iso >. Acesso em: 05 jun. 2017.

12. Fripp J. Ação prática do profissional de cuidados paliativos no domicílio. In: Manual de cuidados paliativos. Academia Nacional de Cuidados Paliativos ANCP. Rio de Janeiro: Diagraphic, 2009.

13. Menezes RA. Em busca da morte: antropologia dos cuidados paliativos. Rio de Janeiro: Garamond: FIOCRUZ, 2004.

14. Sociedade Brasileira de Geriatria e Gerontologia (SBGG). Vamos falar de cuidados paliativos? Brasil, 2015. Disponível em: <http://sbgg.org.br/wp- 
content/uploads/2014/11/vamos-falar-de-cuidados-paliativos-vers--0online.pdf >. Acesso em: 17 mai. 2017.

15. Maciel MGS. Definições e princípios. In: Cuidado Paliativo. Conselho Regional de Medicina do Estado de São Paulo - CREMESP. São Paulo, 2008.

16. Arantes ACLQ. Indicações de cuidados paliativos. In: Manual de Cuidados Paliativos. Academia Nacional de Cuidados Paliativos - ANCP. Rio de Janeiro: Diagraphic, 2009.

17. Maciel MGS. Avaliação do paciente sob cuidados paliativos. In: Manual de cuidados paliativos. Academia Nacional de Cuidados Paliativos - ANCP. Rio de Janeiroi: Diagraphic, 2009.

18. Américo AFQ. As últimas 8 horas de vida. In: Manual de Cuidados Paliativos. Academia Nacional de Cuidados Paliativos - ANCP. Rio de Janeiro: Diagraphic, 2009.

19. Kira CM. As últimas 48 horas. In: Cuidado Paliativo. Conselho Regional de Medicina do Estado de São Paulo - CREMESP. São Paulo, 2008.

20. Taquemori LY, Sera CTN. Interface intrínseca: equipe multiprofissional. In: Cuidado Paliativo. Conselho Regional de Medicina do Estado de São Paulo - CREMESP. São Paulo, 2008.

Recebido: 31 de janeiro de 2018. Publicado: 20 de fevereiro de 2018

Correspondência: Gabriella Belém Vasconcelos. E-mail: gabriella.belem@gmail.com

Conflito de Interesses: os autores declararam não haver conflito de interesses. 